Research Article

\title{
The Influence of Multimedia Input Mode Based on Complex System Discrete Model on College Students' English Ability
}

\author{
Xiaoge Wang $(\mathbb{D}$, Weihong Zhao, and Yuanyuan Xu \\ Qingdao Huanghai University, Qingdao, Shandong 266555, China \\ Correspondence should be addressed to Xiaoge Wang; wangxg@qdhhc.edu.cn
}

Received 6 September 2021; Accepted 12 October 2021; Published 31 October 2021

Academic Editor: Gengxin Sun

Copyright ( $\odot 2021$ Xiaoge Wang et al. This is an open access article distributed under the Creative Commons Attribution License, which permits unrestricted use, distribution, and reproduction in any medium, provided the original work is properly cited.

\begin{abstract}
With the rapid development of the Internet, the amount of data increases sharply. In this network age, data have been generated, such as multimedia, social networks, and search records. Due to the generation of data, complex system discrete model technology is also widely used in all walks of life. Multimedia learning is a popular teaching mode in the world. Through the discrete model technology of the complex system, this paper deeply studies the impact of multimedia input on college students' English ability. Because the modeling and sampling of complex systems are abstract and not fixed, approximation and control methods are adopted in discrete time, which greatly improves the reliability of the final research results. Compared with the original discrete modeling technology, this paper adds data sampling compression and assimilation technology on the basis of the original discrete modeling technology of the complex system. The results show that, by adding the technical application of controllable and observable systems, the discrete model of the whole complex system can reduce the error of the final output data. At the same time, this paper compresses and simplifies the data nodes at the microlevel. Macroscopically, it reduces the scale, especially the number of node data compression. The final result also realizes the data scale and greatly shortens the calculation time. This study has greatly improved the accuracy of the impact of multimedia on Chinese college students' English ability in practical application.
\end{abstract}

\section{Introduction}

The constituent elements of the complex dynamic system are many scientific and technological products [1]. The complexity of the system is mostly reflected in the composition, structure, and scale of the system. This type can be further explored by creating a complex network, for example, computer network, financial system, power system, and transportation system $[2,3]$. The complexity of the system can even be reflected through human social life, such as the multimedia direction studied in [4]. For the research in this field, the difficulty of discrete modeling of complex systems lies in the increase of control nodes and the processing of a large amount of data. It is also a hot issue in the study of this kind of discrete model so far. For the influence between multimedia and college students' English ability, the key purpose of the complex system lies in the two key points of "control system dynamic direction and detection system" and "system data analysis" $[5,6]$. In the two knowledge fields of discrete modeling, control and construction of complex systems, academic researchers who study complex system modeling upload a large number of research results on relevant websites every year. Most mathematicians have been mature in the cognition of complex systems through continuous exploration. In the early stage of research, due to the limitations of the development of science and technology, people can only study with the help of the knowledge of discrete mathematics. Now, with the development of computer and network, the research in this field has made great progress [7]. Through the integration of various technologies by researchers, the knowledge in books has been transformed into today's discrete model of the complex system [8]. However, in fact, it is a very difficult technical problem to realize the connection between nodes and networks and face the huge network scale in the seemingly simple technical principle of mankind [9]. This also indicates that the control of complex systems is the focus of academic researchers. 
Nowadays, some university courses still maintain the traditional multimedia learning and teaching methods and pay more attention to static pictures, such as the impact of PPT combined with the teacher's teaching methods to transfer knowledge to students [10]. Of course, this method has made a great impact in the past five years, but in recent two years, with the rise of film and television and animation multimedia technology, this technology has been widely used in schools. The researcher's questionnaire survey shows that most students can improve their English learning ability through the new multimedia teaching method, but some studies show that it is the opposite result [11]. In order to more systematically verify and predict the overall development trend in the future, the complex system discrete model technology can be used to integrate the data within decades [11]. It can also further correlate graphics, senses, and knowledge capabilities through discrete modeling of complex systems.

This paper is mainly divided into three parts. Section 1 is a brief description of discrete model modeling technology of the complex system and learning multimedia cognitive theory. Section 2 is the research on the influence of multimedia input on college students' English ability under the modeling method based on the discrete model of the complex system, and the research on the control system of multimedia in learning ability under the discrete modeling method of the complex system. The main research content is to add some new methods to the original complex discrete model, add modeling technology, and then carry out experimental verification. Section 3 uses the discrete modeling method of the complex system to analyze the research results and the impact of multimedia input on college students' English ability.

\section{Related Works}

The learning object of multimedia is mainly learners with different characteristics. For learners, there are many factors that will affect the learning effect $[12,13]$. For example, learners' learning interests and their own stored knowledge, ability, experience, motivation, time, etc. $[14,15]$. According to the existing data, the learning effect of multimedia is affected by its own reserved knowledge and experience and personal cognitive ability [16]. This shows that we should focus on learners' personal cognitive ability [17]. Therefore, we should combine the presentation of multimedia with personal cognitive ability. Then, integrate the data through system modeling technology and finally analyze the impact of multimedia on college students' English ability [18]. A large number of existing data on the network are controversial, so we should find the most scientific research methods to compare from many aspects and finally make the most correct research results.

The complex discrete dynamic modeling technology in the United States is very mature, and it is widely used in the financial industry. Universally, such as the rise and fall range of stocks, the daily financial data are discretized, filtered, and regenerated into thermal coding [19]. Finally, the data will be merged, and the rise and fall range of the stock market will naturally become clear at a glance.
Discrete modeling technology is applied in power attributes. The reason for using complex discrete dynamic modeling technology is that the power monitoring system has a large amount of monitoring data and complex types under the background of power big data. The traditional discrete modeling technology cannot meet the current situation $[20,21]$. In order to preprocess the generated data timely and effectively, the complex discrete dynamic modeling technology is applied, which is suitable so far.

Complex discrete dynamic modeling technology is widely used in the automotive industry. It is well known that the automotive industry is very developed [22]. In the production of automobile parts and various devices, a large number of product data need to be measured. In order to clearly see the individual difference of data, discrete dynamic modeling technology is applied. The introduction of this technology makes the data of automobile products and evaluation standards better compared, and the same produced cars are safer.

China has been using discrete dynamic modeling technology for complex systems in aerospace and chip manufacturing [23]. The aerospace field mainly studies various data returned by experimental testing, in order to ensure that the overall data is close to the standard data. This has also greatly improved the success rate of China's rocket launch. According to the various applications in complex system discrete dynamic modeling technology, this paper studies the parameter data based on the technology of complex system discrete dynamic model and the impact of multimedia input on Chinese college students' English ability. In this study, we mainly study the relationship between human learning ability and multimedia teaching methods, so we can only consider the directed complex dynamic discrete model, and the use of the model tends to study the heterogeneity of nodes [24]. Considering the huge amount of dynamic data, the technology of compressing nodes is also used. Finally, based on the summary of the final results based on the complex system, this paper analyzes the impact between college students' English ability and multimedia.

\section{Research on the Influence of Multimedia Input on College Students' English Ability Based on the Complex System Discrete Model}

3.1. Research on Multimedia Output Sampling and Model Simplification of Learning Ability Based on the Discrete Modeling Method of the Complex System. The modeling basis of the complex system is to model the data of learning ability according to the input of multimedia. The modeling process must be carried out together with the design, and part of the network topology information is required, and the whole can be realized digitally. The core is to first select the keywords between multimedia and students' ability, determine the keywords of different types, and simply classify the keywords. The main control technical problems of complex dynamic systems include two points. One reason is that the nature of the network is different from the superposition of 
keywords, and the inrush phenomenon often occurs. The whole part is not as big as the sum of nodes. This also shows that each node is interrelated, and each part is very important, even if it seems small. On the contrary, if the network is nonlinear, a large number of coupling will make the data difficult to analyze. Therefore, the nonlinear model can only exist in a discrete model of a complex system driven by data and keywords between multimedia and learning ability. Based on the above situations, firstly, a simple architecture model between multimedia and students' learning ability is made. The flowchart of the multimedia cognitive theory model is shown in Figure 1.

As can be seen from Figure 1, the whole flowchart represents a knowledge processing flow system. In the multimedia teaching method, it mainly receives knowledge intuitively through the human sensory system. Images and words are the embodiment of vision, and speech is the way of hearing. Listening and writing are two important knowledge bases in college English. Of course, the whole process is in the motivation of learners' active learning, so as to turn knowledge into the embodiment of their own ability. On this basis, the discrete modeling technology is applied. As can be seen from the flowchart, there are many different types of keywords. The following differential equation formula is applied to create a complex discrete model:

$$
\begin{aligned}
\frac{d v i(t)}{d t} & =\varphi_{o i}(v i(t))+\sum_{j=1}^{|v|} A_{i, j} \varphi_{l i}\left(v_{i}(t)\right) \varphi_{2 i}\left(v_{i}(t)\right), \\
v_{i}(k) & :=v_{i}(k \tau)=v_{i}\left(\left|\frac{t}{\tau}\right|\right), \\
u(t) & =u(k \tau):=u(k), \quad \forall t \in[k \tau,(k+1) \tau] .
\end{aligned}
$$

In the first formula, it can be seen that each node exists independently, so the coupling in the node is a complex dynamic network that can be separated. The second and third formulas are mainly the implementation of sampler and holder, which can find and receive input keywords. The received keywords have different structures but belong to related words, so Von Neumann formula is adopted:

$$
n_{n v}=\left\{n_{j}=\left(x_{1 i}, \ldots, x_{d j}\right), x_{k j}=\{-1,0,1\}, k=1, \ldots, d \sum_{k=1}^{d}\left|x_{k j}\right| \leq 1\right\} .
$$

The dynamic discrete modeling technology of the complex system is used between multimedia and students' English ability by putting information and data into the model. Several adjacent data are processed separately and then displayed in the graph. The modeling flowchart of adding data to the model is shown in Figure 2.

Compared with other complex system discrete dynamic modeling technologies, in order to improve the accuracy of research, a data processing method other than "neighbor keyword" is added to the system discrete modeling process. This processing method is actually an ordinary discrete modeling application. The two results can be compared artificially. If the results are close, the data can be further added. Otherwise, it will be discarded and not included in the final statistical results. In the experimental research, data loss events sampled from big data often occur in the experimental research. Once too much data are lost, it will cause some differences in the research results, as shown in Figure 3.

According to the comparison of the two broken lines in Figure 3, the loss of data will affect the overall data trend. If there is a large-scale loss of data, the research results will certainly fail. In order to prevent the result error caused by the lack of data, the robust weight recursive least square algorithm is added to the research method of this paper. The expression is

$$
\begin{aligned}
& \theta_{i}(k)=\underset{\theta \in R^{n i}}{\arg \min }\left\{\frac{1}{2} \omega_{i}^{2}(k)\left(v_{i}(k)-\theta_{i}^{T}(k) \phi_{i}^{2}(k)\right)+\frac{\lambda_{s}}{2} \theta_{i}(k) \theta_{i}^{T}(k-1)\left(\theta_{i}(k)-\theta_{i}(k-1)\right)\right\}, \\
& n_{i}(k)=2+D^{-}\left(v_{i}\right) \\
& \theta_{i}(k)=\left(\alpha_{i 1}(k), \alpha_{i 2}(k), \beta_{i j}(k)\right)^{T} .
\end{aligned}
$$

Define $p_{i}^{-1}(k-1)$ as the formula

$$
p_{i}^{-1}(k)=\lambda_{s} p_{i}^{-1}(k-1)+\omega_{i}^{2}(k) \phi_{i}(k) \phi_{i}^{T}(k) .
$$

Preset $p_{i}^{-1}(0)$ as positive definite value and $0<\lambda_{s}<1$ as the forgetting factor:

$$
\begin{aligned}
\omega_{i}(k) & =\frac{1}{\|\sigma(\kappa)\|}, \\
\sigma^{2}(k) & =\left(v_{i}(k)-\theta_{i}^{T}(k-1) \phi_{i}(k)\right)^{2}+\sigma_{0} .
\end{aligned}
$$

The combination of the above formulas can greatly improve the convergence. The discrete dynamic modeling 


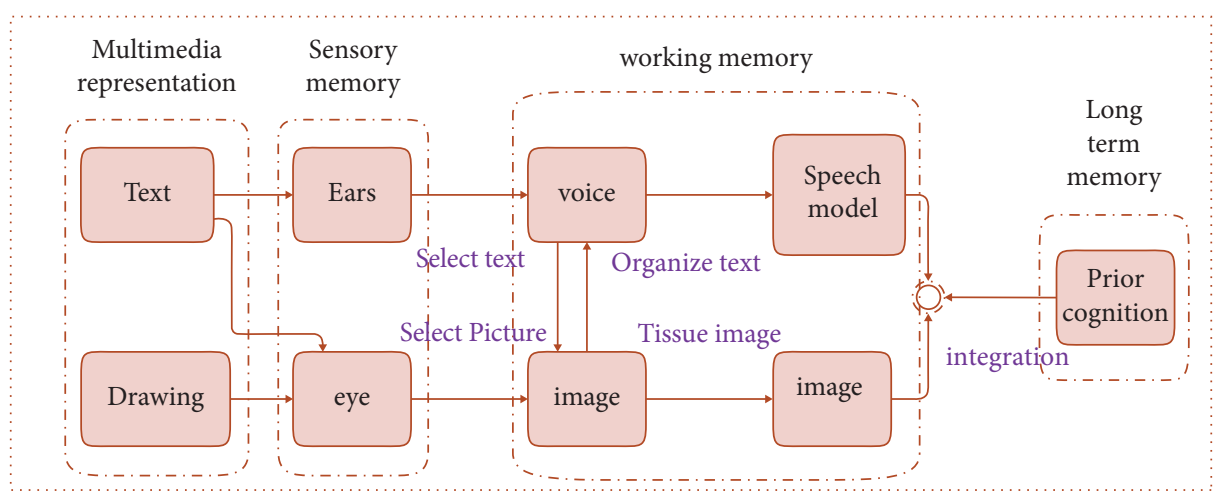

FIgURE 1: Flowchart of the multimedia cognitive theory model.

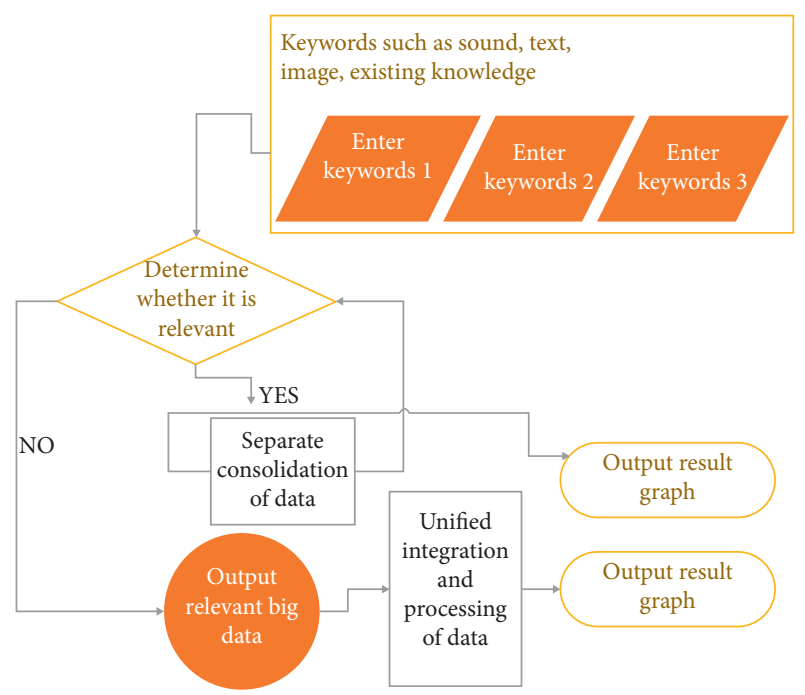

FIGURE 2: Discrete modeling flowchart for input keywords.

theory and least square algorithm based on the complex system use the same time, which has a great correlation with the effectiveness and accuracy of the complex discrete model. Theoretically, if the sampling time is infinitely close to zero, the error in the complex discrete model will be infinitely close to zero. Based on the formula which is a perfect assumption, there is no similar situation in real life. This high-frequency completion rate is difficult to achieve. For the current control design, generally speaking, it is not necessary to pursue the high-frequency sampling rate in the establishment of the model because, to be close to real life, the errors encountered in the research can also be accepted. The comparison error of multiple sampling single analysis is shown in Figure 4.

As shown in Figure 4, the error rate in the three same experiments will not exceed $1 \%$, which is also a basis for ignoring the error. On the whole, it does not bother the experimental results.

When building the discrete model of the complex system, we need to get the complete topology in big data. However, it is not easy to obtain the complete topology and nodes, so a method of compressing nodes is cited, as shown in Figure 5.

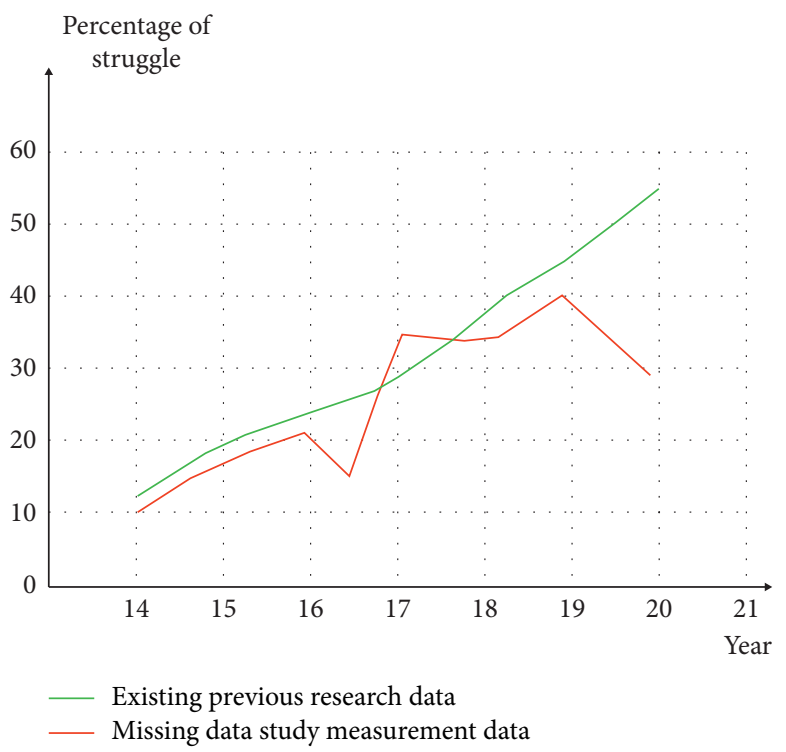

Figure 3: Comparison of existing data results and missing data studies.

As shown in Figure 5, $v_{1}$ and $v_{2}$ and many nodes are finally compressed to only two nodes. Such a compression method is conducive not only to the storage of data but also to the accuracy of research results. The dynamic expression of the compression node is

$$
\begin{aligned}
\frac{d v_{s}(t)}{d t} & =\psi o_{s}\left(v_{s}(t)\right)+\psi 1_{s}\left(v_{s}(t)\right) \bar{\psi} 2_{s}\left(v_{c}(t)\right), \\
\bar{\psi} 2_{s} & =\psi 2_{s}\left(R_{q}^{-1}\right) .
\end{aligned}
$$

The functions in the expression are continuous. After a series of transformations, the nodes disappear. The content in the disappeared node will not disappear, but will be put into the $v_{s}$ node after compression. $v_{s}$ adaptive complex system discrete dynamic model expression is

$$
v_{s}(k+1)=\hat{\alpha}_{s_{1}}(k) v_{s}(k)+\hat{\alpha}_{s_{2}}(k) v_{s}(k-1)+\hat{\beta}(k) v_{c}(k) .
$$




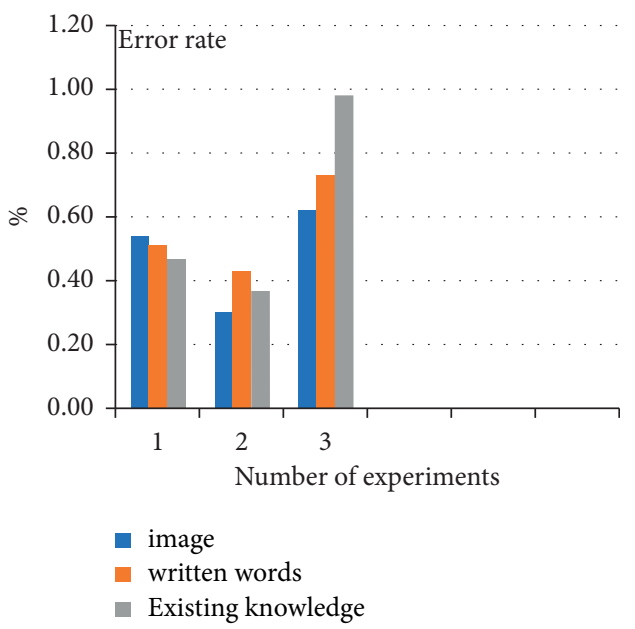

FIGURE 4: Error histogram of three kinds of parameters in three experiments.

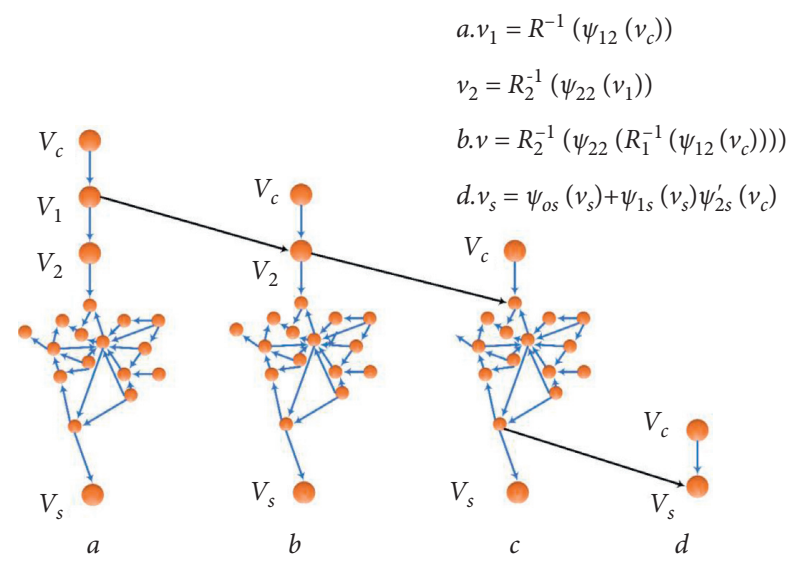

Figure 5: Compressed data node diagram.

From another point of view, it can be assumed that there is an unimpeded channel between nodes and driving nodes. Then, the content of all nodes in the channel can be compressed, and the complex dynamic network scale and model also become simple. The expression of the overall adaptive discrete model of complex network is

$$
v_{s}(k+1)=\hat{A}_{1}(K) v_{s}(K)+\hat{A}_{2}(K) v_{s}(K-1)+\hat{B}(K) v_{c}(k)
$$

Ensure that the most suitable data compression method is selected in the subject studied in this paper, and all kinds of data are put into the built model for comparison, as shown in Figure 6.

As can be seen from Figure 6, the combination of topology and compressed nodes is very effective for the data processing between multimedia and English learning ability studied in this paper. It can compress data in a short time with high efficiency. We store more keyword-related data in the same time to improve the total capacity.

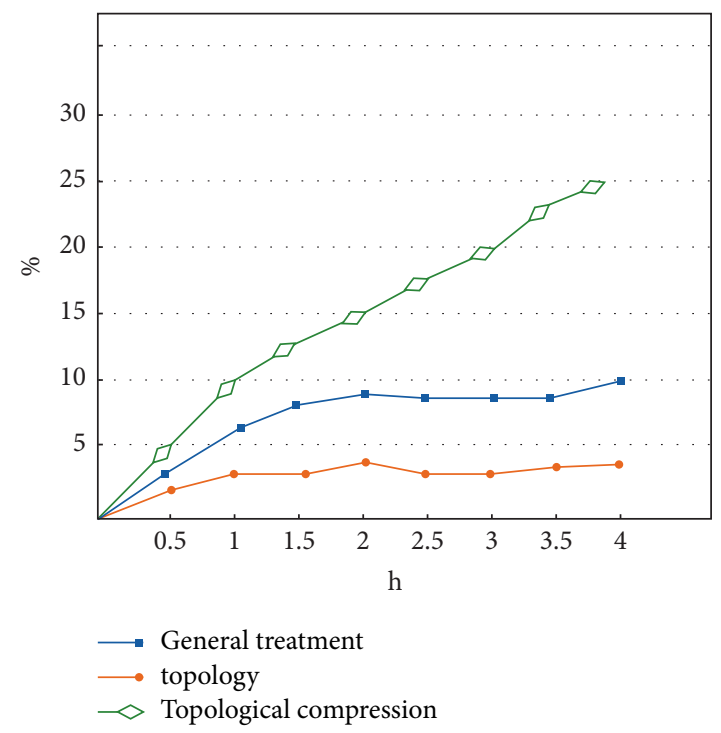

Figure 6: Comparison of processed data under three modes.

3.2. Research on Multimedia Control System for Learning Ability Based on the Discrete Modeling Method of the Complex System. The complex discrete dynamic modeling technology studied in this paper is also a very important part for the stability and control of the whole system. In modeling, the function of over weighing driving node is equivalent to that of controller. The driving node controller is used to model, and a discrete controller is used as the throttle position controller. Create a system driven model. A step signal is used as the input of the system. The delay module and discrete-time integration module are used: the input and output realized by the two methods are viewed in the same oscilloscope by using the reverse integration method. Finally, the discrete state function model is created by defining module parameters with different names. The driving node can control the dynamic network part of the whole complex discrete dynamic modeling. The driving node is usually located at the first 
starting node. The influence driving node can be driven by artificial external stimulation. The dynamic equation is

$$
\frac{d v_{i}(t)}{d t}=\psi o_{i}\left(v_{i}(t)\right)+\sum_{l=1}^{L} B_{i, l} u_{l}(t)
$$

Input the external stimulus behavior, and take the input signal as one of the nodes. At this time, the dynamic expression of the observation node is

$$
v_{s}(k+1)=\hat{\alpha}_{s 1}(k) v_{s}(k)+\hat{\alpha}_{s 2}(k) v_{s}(k-1)+\sum_{l=1}^{L} \beta_{i, l}(k) u_{l}(k)
$$

Once the input signal has an impact on the nodes in the data, it can be proved that the stimulation signal node is connected with the driving node. In this way, in the discrete dynamic modeling process of the whole complex system, the characteristic model formula of the observation node is

$$
v_{s}(k+1)=\hat{A}_{1}(k) v_{s}(k)+\hat{A}_{2}(k) v_{s}(k-1)+\hat{B}(k) u(k)
$$

After constructing the nodes of stimulation signals, the whole complex discrete modeling system can control the minimum amount of external human input. At the technical level of control, there is another kind called traction control. In short, the purpose of this control is to make all nodes in the data reach a consistent state. It is very important to synchronize the whole system through the traction controller. The traction controller is applied to the research in this paper, and the synchronization data track state of each node is obtained, as shown in Figure 7.

As shown in Figure 7, different nodes in a large amount of data have reached a synchronization state and finally come together. The construction formula of the tractor is

$$
u_{i}(k)=k \frac{u(k)-\theta_{i}^{T}(\bar{K}-1) \phi_{i}(k)}{\beta_{i}(\hat{k})+\lambda_{0}} .
$$

The main purpose of this formula is to expect the values of all nodes to reach a stable state. Moreover, the traction controller is realized according to the digital signal sampled from the data output of the node. The traction controller based on the discrete model of the complex system is also a complex controller, which can control the whole process of discrete dynamic modeling. The controller can also be used for control design and split analysis of the overall network structure. The model formula of traction controller is as follows:

$$
\begin{aligned}
& v_{i}(k+1)=f_{2 i} v_{i}(k)+\sum_{j=1}^{|v|} A_{i j} \beta_{i j} v_{j}(k)+\beta_{l} u_{l}(k), \\
& v_{i}(k+1)=f_{2 i} v_{i}(k)+\sum_{j=1}^{|v|} A_{i j} \beta_{i j} v_{j}(k) .
\end{aligned}
$$

By adding the true value which can distinguish whether the data parameters converge or not, the whole complex

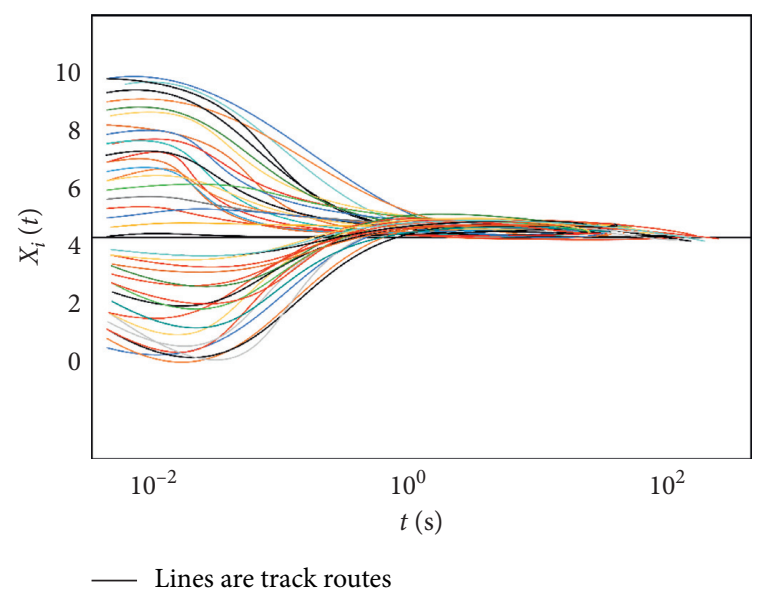

FIGURE 7: Data diagram after using tractor between different nodes.

traction controller is locally asymptotic and stable in the existing discrete model. In order to verify the function of the tractor, a data input is carried out. The signal frequency of the data is shown in Figure 8.

As shown in Figure 8, after two different types of numerous data inputs, all the overall data signals are close to a balanced and stable state. This also shows the function of the complex traction controller.

\section{Analysis of the Impact of Multimedia Input on College Students' English Ability under the Modeling Method Based on the Complex System Discrete Model}

4.1. Analysis of Research Results of Multimedia Output Sampling and Model Simplification of Learning Ability Based on the Discrete Modeling Method of the Complex System. The discrete modeling of the whole complex system is based on the influence of multimedia input on college students' English ability. A large amount of data from the network is put into the model for systematic testing. Based on the discrete dynamic modeling technology, in order to avoid data loss, a robust weight recursive least square algorithm is added. The combination of topology and compression node is also added to store more data information and output information in the effective time. Firstly, the keywords related to college students' English learning ability and multimedia are input into the complex system discrete model. The input data include the same type of data and different types of data. After data input, the data will be protected to avoid data loss, and then, different types of data will be homogenized. Finally, the data are node compressed to read more data for research. In this experiment, a total of 4000 data are sampled, and the whole system based on the above technologies is tested under different large-scale data. The test results are shown in Figure 9.

It can be seen from Figure 9 that the data processing rate of the discrete dynamic model of the complex system for sampling is also different under the data of different scales. The overall rate does not exceed one percent. In the face of a large amount of data, the system model can still maintain a 

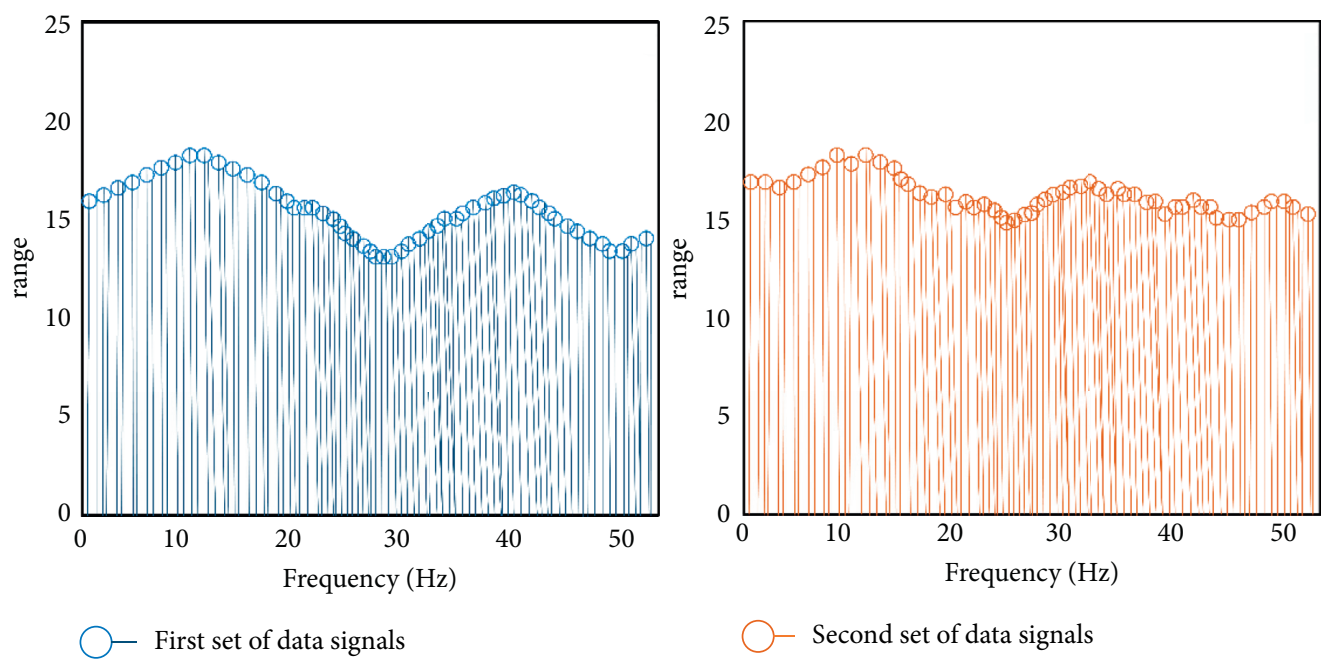

Figure 8: Frequency distribution diagram of two groups of data signals.

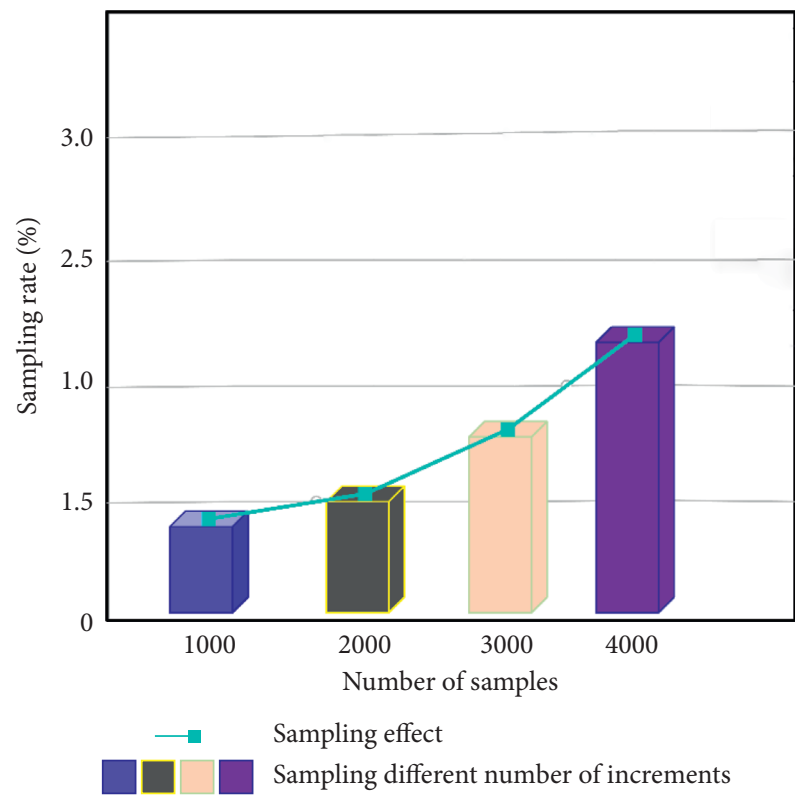

FIGURE 9: Large-scale different data sampling and processing rate diagram.

certain processing speed. This also proves that, after the modeling technology is based on the above algorithms, the processing capacity of the whole system for sampling data has reached a very fast speed. The fast processing of sampled data is very beneficial to the influence between multimedia and college students' learning ability. The data processing rate is high, and it is also very suitable for many modern industries. Moreover, only based on large-scale data can we make statistics and predict the correlation between multimedia and college students' English ability.

\subsection{Analysis of Research Results of Multimedia Control System} for Learning Ability Based on the Discrete Modeling Method of the Complex System. After solving the same normalization of sampling data and data compression, the output data is put into the complex discrete model system. By adding an

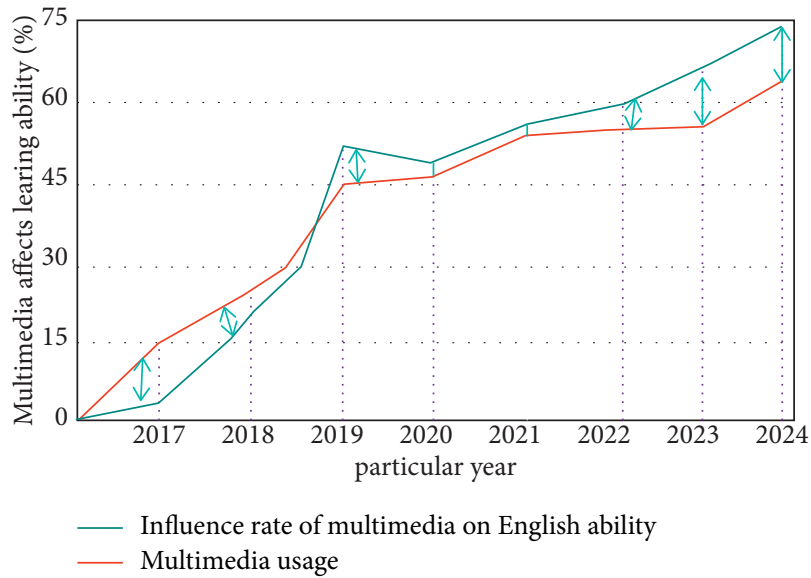

FIGURE 10: Influence of multimedia on learning and multimedia application rate diagram.

artificial external stimulus point to the system to affect the driving node of the starting data, the observation node can be added after the two signals have an impact. In this way, the state of the discrete model can be stabilized artificially. The traction controller is also added so that each part of the whole complex discrete dynamic model can be stably executed without accidental data differentiation. In the implementation of the experiment, firstly, the sampled data affected by relevant multimedia and students' English are assimilated and compressed for data output. The output data can enter the coupling of large-scale data under the discrete model of the complex system, and the traction controller is added to the coupling to stabilize the whole system. Finally, the data are integrated and presented. This experiment is designed to test the impact of multimedia input on college students' English ability and the use of multimedia in recent five years. The results of the test study are shown in Figure 10.

As shown in Figure 10, the English ability of Chinese college students has been improved after multimedia input. Even in the next three years, through systematic data analysis, Chinese college students' English ability will 
improve faster. The use of multimedia in the classroom is also growing. In the model, by controlling the stability of all aspects of the system, the final result of the analysis is also in line with the reality, which also improves the success rate of the whole test research.

\section{Conclusion}

In today's society, complex system discrete model technology is a popular technology in the fields of aviation, finance, medicine, automobile, and so on. Because the data and requirements of each application field are different, many kinds of formulas and algorithms have been added. Therefore, there are many ideas to deal with discrete dynamic modeling of various complex systems. Compared with the original discrete modeling technology, this paper adds the technology of data sampling compression and assimilation to the original discrete modeling technology of the complex system. Two important technologies, controllable and observable, are added to the model. In contrast, the original ordinary discrete modeling cannot transform the complex network system with a large amount of data into a multidimensional unified single system. The results show that, by adding the technical application of controllable and observable systems, the discrete model of the whole complex system can reduce the error of the final output data. In contrast, if the ordinary discrete modeling technology is directly applied to the dynamic modeling technology of complex discrete systems, it will only cause the disorder of data and systems. From the structure of the whole complex discrete system, whether it is a control system or an observation system, the data information generated by not all nodes is valuable. Therefore, the complex system controller is introduced to observe the output node data. In general, there is no common system that can compress the node data. In contrast, this paper compresses and simplifies the data nodes on the microlevel. Macroscopically, it reduces the scale, especially the number of node data compression. The final result also realizes the data scale and greatly reduces the calculation time. In the study of the impact between multimedia and Chinese college students' English ability, the results show that it has greatly improved the accuracy. However, the research of this paper lacks the verification analysis of the proposed model. Therefore, the content of this part needs further research.

\section{Data Availability}

The research data used to support the findings of this study are included within the article.

\section{Conflicts of Interest}

The authors declare that they have no conflicts of interest.

\section{References}

[1] L. Shen, L. G. Di, D. Zhu et al., "Mechanical responses of steel fiber-reinforced concrete after exposure to high temperature: experiments and mesoscale discrete modeling," Journal of Engineering Mechanics, vol. 147, no. 11, 2021.
[2] T. D. Mezebish, R. B. Chandler, G. H. Olsen et al., "Wetland selection by female ring-necked ducks (Aythya collaris) in the Southern atlantic flyway," Wetlands, vol. 41, no. 6, 2021.

[3] Z. W. Chen and X. P. Li, "Designing corridor systems with modular autonomous vehicles enabling station-wise docking: discrete modeling method," Transportation Research Part E, vol. 152, 2021.

[4] T. Loossens, F. Tuerlinckx, and S. Verdonck, "A comparison of continuous and discrete time modeling of affective processes in terms of predictive accuracy," Scientific Reports, vol. 11, no. 1, p. 6218, 2021.

[5] M. Fukushi, C. Guevara, and M. S. Angelo, "A discrete choice modeling approach to measure susceptibility and subjective valuation of the decoy effect, with an application to route choice," Journal of Choice Modelling, vol. 38, 2021.

[6] B. Gilberto, P. Andrea, S. Davide, L. Franz, T. Sara, and B. Raffaele, "Integration of photogrammetry from unmanned aerial vehicles, field measurements and discrete fracture network modeling to understand groundwater flow in remote settings: test and comparison with geochemical markers in an Alpine catchment," Hydrogeology Journal, vol. 29, 2021.

[7] G. Ahmadreza, M. Ahmad, and N. Ali, "Hybrid continuousdiscrete modeling of an ordinary stone column and micromechanical investigations," Geotechnical and Geological Engineering, vol. 29, 2021.

[8] D. E. Stewart, D. W. Wood, J. B. Alcorn et al., "A revealed preference analysis to develop composite scores approximating lung allocation policy in the US," BMC Medical Informatics and Decision Making, vol. 21, no. 1, p. 8, 2021.

[9] J. C. Quezada and G. E. Villavicencio, "Discrete modeling of waste rock dumps stability under seismic loading," EPJ Web of Conferences, vol. 249, 2021.

[10] H. B. Ye, F. Xiao, and H. Yang, "Day-to-day dynamics with advanced traveler information," Transportation Research Part $B$, vol. 144, 2021.

[11] D. A. Edwards, B. Emerick, A. G. Kondic, K. Kiradjiev, C. Raymond, and M. Zyskin, "Mathematical models for the effect of anti-vascular endothelial growth factor on visual acuity," Journal of Mathematical Biology, vol. 81, pp. 1397-1428, 2020.

[12] I. Edouard, B. H. Haithem, and V. V. John, "High-stress impact-abrasion test by discrete element modeling," Computational Particle Mechanics, 2020.

[13] A. A. Yu, Y. A. Antokhina, V. M. Balashov, E. G. Semenova, and A. G. Varzhapetyan, "Computer simulation of processes in technical systems," Journal of Physics: Conference Series, vol. 1691, no. 1, 2020.

[14] Y. Guo, Q. Chen, Y. Xia, T. Westover, S. Eksioglu, and M. Roni, "Discrete element modeling of switchgrass particles under compression and rotational shear," Biomass and Bioenergy, vol. 141, 2020.

[15] A. Abramian, L. Staron, and P. Y. Lagrée, "The slumping of a cohesive granular column: continuum and discrete modeling," Journal of Rheology, vol. 64, no. 5, pp. 1227-1235, 2020.

[16] J. Zhi and T. E. Tay, "Interrogating failure mechanisms of notched composites through a discrete crack modeling approach," Composites Science and Technology, vol. 196, 2020.

[17] L. T. Wen, Q. Y. Sheng, and H. H. Yan, "Discrete element modeling on crack evolution behavior of sandstone containing two oval flaws under uniaxial compression," Arabian Journal of Geosciences, vol. 13, no. 1, 2020.

[18] J. William and B. Francois, "Discrete element models of fracture in tooth enamel: failure mode competition and statistical effects," Journal of the Mechanics and Physics of Solids, vol. 137, 2020. 
[19] H. J. Van, E. A. Audenaert, R. Atkins Penny, and A. E. Anderson, "A combined geometric morphometric and discrete element modeling approach for hip cartilage contact mechanics," Frontiers in Bioengineering and Biotechnology, vol. 8, 2020.

[20] C. Sergey, V. Ilia, K. Iuliia, T. Nikita, and N. Gleb, "Empirical study on discrete modeling of urban intersection management system," International Journal of Embedded and Real-Time Communication Systems (IJERTCS), vol. 11, no. 2, 2020.

[21] Z. Wenbo, D. D. Tannant, C. Xiaojun, X. Cong, and H. Xinli, "Improved discrete element modeling for proppant embedment into rock surfaces," Acta Geotechnica, vol. 15, no. 2, 2020.

[22] B. Hicham, A. Abdallah, M. Yann, B. Matthieu, and D. Laurent, "Discrete element modeling of concrete under high stress level: influence of saturation ratio," Computational Particle Mechanics, vol. 8, 2020.

[23] V. Marzulli, C. L. A. Torres, L. A. Di et al., "Impact on granular bed: validation of discrete element modeling results by means of two-dimensional finite element analysis," Granular Matter, vol. 22, no. 1, 2020.

[24] J. Aditya, S. Claire, F. Jürgen, and B. G. E. Bogin, "Discrete modeling of a longwall coal mine gob for CFD simulation," International Journal of Mining Science and Technology, vol. 30 , no. $4,2020$. 